\title{
From cyanobacteria to plants: conservation of PII functions during plastid evolution
}

\author{
Vasuki Ranjani Chellamuthu • Vikram Alva • \\ Karl Forchhammer
}

Received: 3 August 2012/ Accepted: 26 October 2012/Published online: 29 November 2012

(c) Springer-Verlag Berlin Heidelberg 2012

\begin{abstract}
This article reviews the current state-of-the-art concerning the functions of the signal processing protein PII in cyanobacteria and plants, with a special focus on evolutionary aspects. We start out with a general introduction to PII proteins, their distribution, and their evolution. We also discuss PII-like proteins and domains, in particular, the similarity between ATP-phosphoribosyltransferase (ATP-PRT) and its PII-like domain and the complex between $\mathrm{N}$-acetyl-L-glutamate kinase (NAGK) and its PII activator protein from oxygenic phototrophs. The structural basis of the function of PII as an ATP/ADP/ 2-oxoglutarate signal processor is described for Synechococcus elongatus PII. In both cyanobacteria and plants, a major target of PII regulation is NAGK, which catalyzes the committed step of arginine biosynthesis. The common principles of NAGK regulation by PII are outlined. Based on the observation that PII proteins from cyanobacteria and plants can functionally replace each other, the hypothesis that PII-dependent NAGK control was under selective pressure during the evolution of plastids of Chloroplastida and Rhodophyta is tested by bioinformatics approaches. It is noteworthy that two lineages of heterokont algae,
\end{abstract}

A contribution to the Special Issue on Evolution and Biogenesis of Chloroplasts and Mitochondria.

V. R. Chellamuthu · V. Alva

Department of Protein Evolution, Max Planck Institute

for Developmental Biology, Spemannstrasse 35,

72076 Tübingen, Germany

e-mail: vasuki-ranjani.chellamuthu@tuebingen.mpg.de

V. R. Chellamuthu · K. Forchhammer $(\square)$

Interfakultäres Institut für Mikrobiologie und Infektionsmedzin der Eberhard-Karls-Universität Tübingen,

Auf der Morgenstelle 28, 72076 Tübingen, Germany

e-mail: karl.forchhammer@uni-tuebingen.de diatoms and brown algae, also possess NAGK, albeit lacking PII; their NAGK however appears to have descended from an alphaproteobacterium and not from a cyanobacterium as in plants. We end this article by coming to the conclusion that during the evolution of plastids, PII lost its function in coordinating gene expression through the PipX-NtcA network but preserved its role in nitrogen (arginine) storage metabolism, and subsequently took over the fine-tuned regulation of carbon (fatty acid) storage metabolism, which is important in certain developmental stages of plants.

Keywords Chloroplast - CLANS · NAGK · Oxygenic phototroph · PII signaling - Synechococcus elongatus

\author{
Abbreviations \\ ATP-PRT ATP-phosphoribosyltransferase \\ NAGK $\quad N$-acetyl-L-glutamate kinase \\ 2-OG 2-Oxoglutarate
}

Introduction to PII signal processors: general
properties and evolution of canonical PII proteins

PII proteins constitute a superfamily of the most widely distributed signaling proteins in nature, represented in all domains of life (Sant'Anna et al. 2009; Huergo et al. 2012). Members of this superfamily are present in almost all taxonomic groups of bacteria and are ubiquitous in nitrogen-fixing methanogens of the archaeal kingdom; however, in eukaryotes they are only found in oxygenic phototrophs (Arcondéguy et al. 2001; Forchhammer 2008). In all cases studied so far, PII proteins are involved in the control of anabolic nitrogen metabolism. They detect the metabolite 
state of the cell by interdependent binding of ATP and 2-oxoglutarate (2-OG) or ADP in a highly conserved manner, and thereby regulate the activity of transcription factors or key metabolic enzymes (Fokina et al. 2010a, b; Truan et al. 2010; Litz et al. 2011; Radchenko and Merrick 2011; Zeth et al. 2012). Interestingly, these PII-regulated target proteins are distinct in different phylogenetic groups of organisms.

Based on the widespread occurrence of the PII superfamily member GlnK in diverse prokaryotes and its conserved genetic coupling with the ammonium transport protein AmtB, it has been hypothesized that modern trimeric PII proteins may have arisen from an ancient trimeric PII protein that originated early in the evolution of prokaryotes in conjunction with the trimeric ammonium transporters to control ammonium uptake in response to the metabolite state of the cells (Thomas et al. 2000; Sant'Anna et al. 2009). Other PII paralogues, GlnB and NifI, may have evolved subsequently from this primordial GlnK protein by gene duplication and functional diversification. These paralogues are implicated in the regulation of nitrogen-dependent gene expression, in the activity regulation of glutamine synthetase, and in the control of nitrogen fixation through a stupendous variety of mechanisms (Huergo et al. 2012; Leigh and Dodsworth 2007; Luque and Forchhammer 2007; Masepohl and Forchhammer 2007).

In cyanobacteria, PII proteins are present in all known species. While most cyanobacteria harbor one PII protein, some strains encode a second or even a third paralogue (Laichoubi et al. 2011). In contrast to many bacteria, where PII proteins (mainly of the GlnB subfamily) are involved in regulation of glutamine synthetase at various levels (Ninfa and Atkinson 2000; Leigh and Dodsworth 2007), cyanobacterial PII proteins have evolved to regulate the ornithine pathway, which leads to arginine and polyamine synthesis, and to the modulation of nitrogen-dependent transcription. In eukaryotes, PII homologues have only been identified in Chloroplastida (green algae and land plants), where they are nuclear-encoded, and in Rhodophyta, where they are coded by the plastid genome (Uhrig et al. 2009). In both these groups, PII is localized in the chloroplast (Hsieh et al. 1998; Ermilova et al. 2012) and appears to control the key step in arginine synthesis, as in cyanobacteria.

\section{PII-like proteins: witnesses of a widely distributed signal processing mode}

In the phylogenetic analysis carried out by Sant'Anna et al. (2009), a novel group of PII-like proteins, termed PII-new group (PII-NG), was identified and included in the PII superfamily based on sequence similarity. These sequences however lack the two PROSITE signatures characteristic of PII proteins (http://prosite.expasy.org). One of these PROSITE patterns (PS00496; Nitrogen regulatory protein P-II, uridylation site 46-51) is not highly conserved even among canonical bacterial PII proteins; it is located in the flexible T-loop region and is subjected to covalent modifications. The second PROSITE signature (PS00638; [ST]$\mathrm{x}(3)-\mathrm{G}-[\mathrm{DY}]-\mathrm{G}-[\mathrm{KR}]-[\mathrm{IV}]-[\mathrm{FW}]-[\mathrm{LIVM}]-\mathrm{x}(2)-[\mathrm{LIVM}])$ is part of the nucleotide binding site (Xu et al. 1998) and is intimately involved in signal perception by PII proteins. Therefore, especially the lack of this latter site indicates that these proteins cannot fulfill canonical PII functions as previously described. Intriguingly, however, the PII-NG encoding genes are localized next to heavy metal efflux pumps and thus might be involved in the regulation of these transporters. The architectural principle of PII proteins seems to be apparently even more widely distributed. It is seen in proteins that do not share appreciable sequence conservation with canonical PII, but have structures that are highly similar to the PII core architecture, except for the loops, which seem to be characteristic for the canonical PII protein. These proteins form a widespread superfamily of trimeric proteins with potential regulatory roles and occur in almost all known organisms (Kinch and Grishin 2002; Arnesano et al. 2003; Saikatendu et al. 2006). While in most cases their function is unknown, in some cases they are known to be involved in diverse functions such as copper tolerance (Arnesano et al. 2003) or anchoring acetylcholinesterase in mammalian neurons (Perrier et al. 2000). Whether these proteins are evolutionary related to PII proteins or not remains to be clarified. A protein domain that exhibits a structure highly similar to PII has been found in the enzyme ATP-phosphoribosyltransferase (ATP-PRT) from Mycobacterium tuberculosis (Cho et al. 2003) and Escherichia coli (Lohkamp et al. 2004). ATPPRT is the first enzyme of the histidine pathway and is allosterically inhibited by AMP and histidine. The C-terminal PII-like domain comprises the binding site for the allosteric inhibitor histidine. The structure of the PII-like domain of ATP-PRT has striking similarities with the PII in complex with the key enzyme of arginine synthesis, $\mathrm{N}$-acetyl-L-glutamate kinase (see structure comparison in Fig. 1). Note that the orientation of the PII-like domain is upside down compared to Synechococcus elongatus PII bound to NAGK. This similarity is highly intriguing and indicates that the PII-like domain is possibly a relic of an ancestral PII-like protein found in ATP-PRT. At present, the PII-like domain functions as a sensory device for ATPPRT, using the architectural principle of PII proteins as signal processing units. Regulation of amino acid biosynthesis reactions (glutamine, arginine, and histidine) thus emerges as a common basis of the function of PII and PIIlike proteins. 


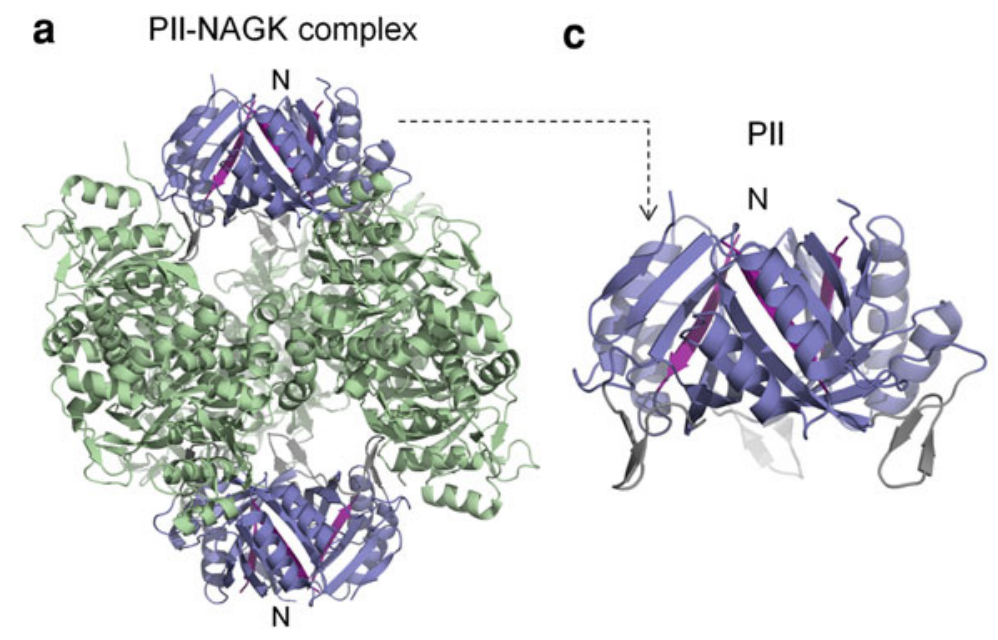

e

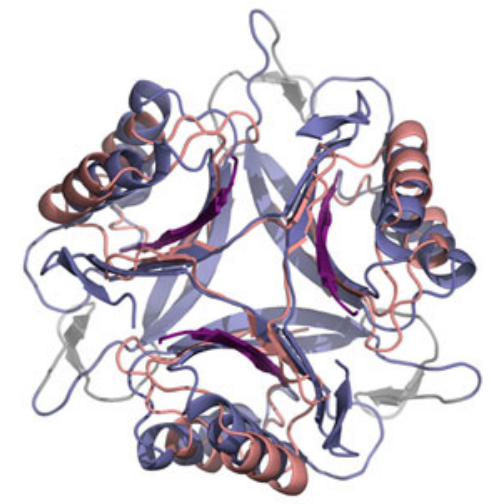

b

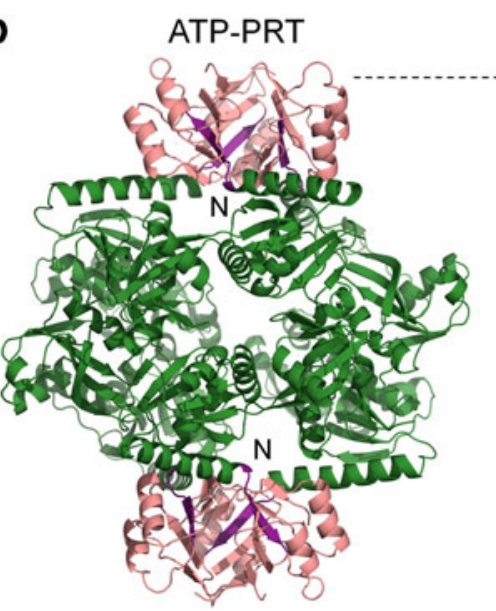

d

f

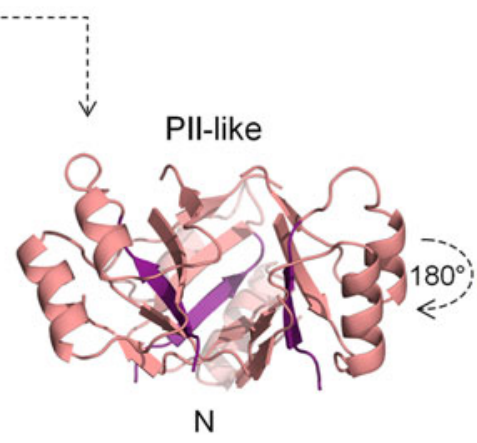

$\mathrm{N}$

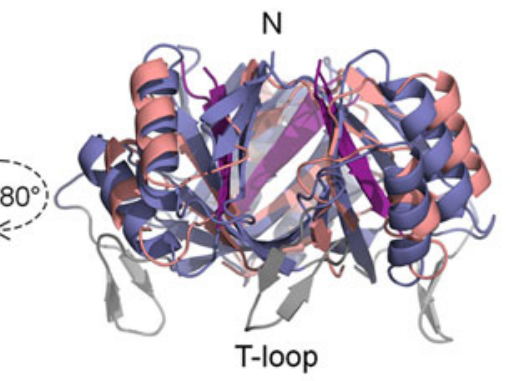

Fig. 1 Comparison of the structures of ATP-phosphoribosyltransferase and PII-NAGK complex. a The complex of PII (slate) and the hexameric $N$-acetyl-L-glutamate-kinase (light green) from $S$. elongatus (PDB ID: 2V5H) are shown. b In the structure of ATP-PRT (PDB ID: 1Q1K) from E. coli, the enzymatic domain is colored in green and the C-terminal, PII-like domain in salmon. c, d Enlarged

views of the PII and PII-like domain are shown in the same orientation as in panels (a) and (b). The N-terminal $\beta$-strands are colored in magenta. e, $\mathbf{f}$ The structural superimposition of the PII and PII-like domain, in top and side view, is depicted. The large T-loops of PII, which are absent in the PII-like domain, are colored in gray. This figure was generated using PyMOL (www.pymol.org)

\section{The PII architecture: from structure to function}

PII proteins have a common architecture: a typical bacterial PII protein consists of three identical subunits of 112 amino acids, which fold into a $\beta 1-\alpha 1-\beta 2-[$ T-loop] $-\beta 3-\alpha 2-\beta 4-$ $\beta 5$ structure. The three subunits assemble with the $\beta$-sheets in the center, forming an intertwined triangular core of three extended $\beta 2$-sheets that pair with the neighboring $\beta 2^{\prime}$ - and $\beta 2^{\prime \prime}$-sheets (Fig. 1e). The C-terminal $\beta 5$-sheet swaps to the $\beta 4$-strand of the neighboring subunit to form part of the active site (Cheah et al. 1994; Leigh and Dodsworth 2007; Forchhammer 2008). The periphery is made up of six (three times two) $\alpha$-helices and nine (three times three) loop regions, which connect the secondary structural elements, to form a barrel-like structure with three lateral clefts between the subunits. For the function of

PII proteins, the three signal transducing T-loops (one per subunit) are of particular importance, since they transmit information on the ligand-binding state of PII into conformational change and mediate many (but not all) of the PII-receptor interactions. The T-loop is a large solventexposed and flexible loop that extends between the $\beta 2$ - and $\beta 3$-strand and protrudes from the bottom of the intersubunit cleft. The three clefts arrange the binding sites for the effector molecules. About 20 structures of PII proteins (Huergo et al. 2012) in complex with ATP or ADP have been deposited in the PDB, including PII from cyanobacteria and Arabidopsis. Comparison of these structures reveals a highly conserved adenyl nucleotide binding mode (Xu et al. 1998; Zeth et al. 2012) with ATP and ADP competing for the same site. The nucleotide is bound essentially through positively charged residues and 


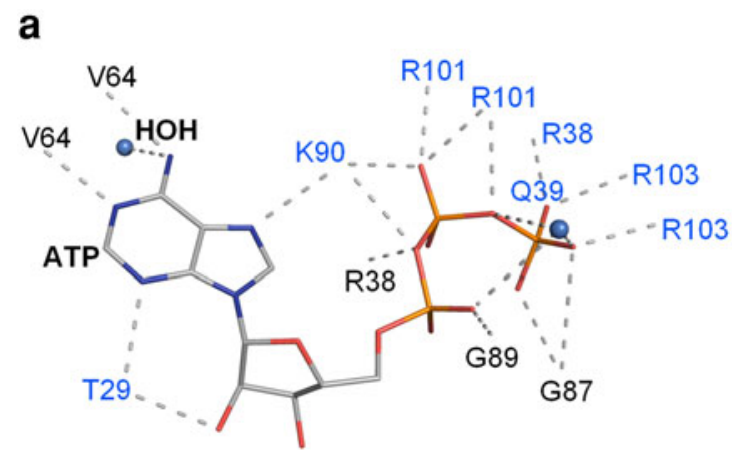

Fig. 2 Comparison of all residues and water molecules that form polar contacts with ATP alone and ATP ligated to 2-OG in PII protein from $S$. elongatus (PDB ID: 2XZW). Amino acids forming contacts with the side-chains are indicated in blue letter code, amino acids

hydrogen bonding contacts contributed from two opposing subunits of the cleft (Fig. 2a): from one subunit, Arg38 and Gln39 residues (belonging to the basal part of the T-loop) contact the $\gamma$-phosphate of ATP, whereas Lys90 and Gly89 contact the $\beta$ - and $\alpha$-phosphate; from the opposite subunit, two Arg residues (101 and 103) of the C-loop contact $\gamma$ - and $\beta$-phosphates, whereas Thr29 and Val64 coordinate the adenosine moiety. The mode of 2-oxoglutarate binding to PII has been clarified recently by solving the crystal structures of PII proteins with Mg-ATP and 2-OG from the archaeon Archaeoglobus fulgidus (Maier et al. 2011), the proteobacterium Azospirillum brasiliense (Truan et al. 2010), and the cyanobacterium S. elongatus (Fokina et al. 2010a). In the latter case, a snapshot of the sequential anticooperative binding of 2-OG to the three sites could be obtained. In all cases, a $\mathrm{Mg}^{2+}$ ion bound by the $\beta$ - and $\gamma$-phosphate of ATP plays an essential role by coordinating the 1-carboxy- and carbonyl-oxygens of 2-OG (Fig. 2b). Furthermore, a universally conserved Lys 58 residue forms a salt bridge with the 5-carboxy-group of 2-OG. The basal part of the T-loop wraps around the 2-OG molecule mainly through backbone interactions of Gln39, Lys40, and Gly41. This results in a unique conformation of the T-loop, thereby affecting the T-loop-mediated protein interactions. From the plant kingdom, only the structure of Arabidopsis thaliana PII has been resolved to date. Overall, its structure corresponds to the archetypical PII architecture described above. However, A. thaliana PII contains N- and C-terminal extensions of 13 and 15 amino acids length, respectively. These extensions are also found in all other plant PII proteins analyzed so far, including green algae, with an exception of chloroplast-encoded PII of red algae (Uhrig et al. 2009; Ermilova et al. 2012). In the A. thaliana structure, the $\mathrm{N}$-terminal extension is organized opposite to the T-loop, whereas the C-terminal extension folds back

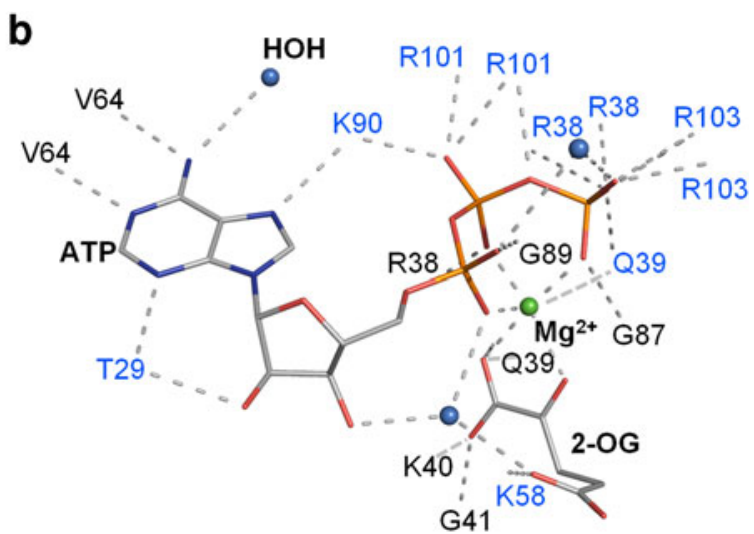

forming backbone interactions are indicated in black. a Polar contacts formed by ATP when bound to PII protein, b ATP undergoes minor changes in conformation to establish polar contacts with residues to enable the 2-OG molecule to ligate in the presence of ATP and $\mathrm{Mg}^{2+}$

towards the effector binding site and in the ATP-free structure, it occupies part of the ATP binding site (Mizuno et al. 2007). It was suggested that these additional sequence elements could mediate plant-specific functions. The effector molecule binding properties of A. thaliana PII are quite similar to those of cyanobacterial PII proteins (Forchhammer and Hedler 1997; Smith et al. 2003; Fokina et al. 2010b): ATP and ADP compete for the same binding site and 2-OG binds in synergy with ATP. However, in case of PII from S. elongatus, ADP does not support the binding of 2-OG, and antagonizes 2-OG binding even in the presence of ATP (Fokina et al. 2011); in contrast ADP does support the binding of 2-OG to A. thaliana PII. Curiously however, 2-OG lowered the affinity of ADP for A. thaliana PII, indicating unfavorable ligand contacts. The unique C-terminal segment, which contacts the effector molecule binding site, might cause these differences. However, ligand-binding properties of other plant PII proteins should be analyzed before generalizing the properties of A. thaliana to all plants.

\section{Modification of PII proteins}

The E. coli GlnB protein was the first PII protein to be carefully studied. As in many other proteobacteria, E. coli PII proteins (GlnB and GlnK) are subject to covalent UMPmodification (uridylylation) at Tyr51, located at the apex of the T-loop (Adler et al. 1975; Atkinson et al. 1994). In fact, uridylylation was long regarded as the hallmark of PII signaling (Arcondeguy et al. 2000; Ninfa and Atkinson 2000). Uridylylation is brought about by the bifunctional enzyme GlnD (Uridylyltransferase/uridylyl-removase), whose activity is regulated by glutamine, with low glutamine levels favoring PII uridylylation and high glutamine 
levels favoring PII deuridylylation. By these means, the PII protein becomes a highly sensitive transmitter of the cellular glutamine status (Jiang and Ninfa 2011). However, uridylylation is not a general trait of PII signaling. The PII protein in the cyanobacterium S. elongatus was shown to be phosphorylated at Ser49, a position adjacent to the uridylylation site of E. coli PII (Forchhammer and Tandeau de Marsac 1994). In vivo, the phosphorylation status of PII depends on the carbon/nitrogen supply of the cells: nitrogen-limiting conditions favor PII phosphorylation, excess nitrogen, preferably in the form of ammonia, causes PII dephosphorylation. With $S$. elongatus cell-free extracts, phosphorylation could be achieved in the presence of millimolar concentrations of 2-OG and ATP. The PII kinase is still unknown; however, the phosphatase of PII-P, PphA, could be identified in Synechocystis PCC 6803 (Irmler and Forchhammer 2001) and has since then been thoroughly studied (Ruppert et al. 2002; Kloft and Forchhammer 2005; Schlicker et al. 2008; Su et al. 2011; Su and Forchhammer 2011, 2012). PphA is a Ser/Thr phosphatase of the PP2C family and the crystal structure of the PphA homologue from the thermophilic cyanobacterium Thermosynechococcus has been solved (Schlicker et al. 2008). In addition to the binuclear metal center, a third metal, which is occasionally observed in bacterial PP2C homologues, was shown to be an essential part of the catalytic center (Su et al. 2011). Recognition of the phosphorylated PII protein involves a flap subdomain, which shields the catalytic center of PphA (Su and Forchhammer 2011). The formation of a precisely fitted substrate-enzyme complex is a prerequisite for dephosphorylation. The conformation of the T-loop plays a critical role in this process: only when PII is non-ligated with 2-OG, PphA is able to dephosphorylate PII. As long as PII-P resides in the Mg-ATP/2OG ligated state, it is protected from dephosphorylation (Ruppert et al. 2002; Su and Forchhammer 2011), implying that the 2-OG induced conformation of the T-loop does not fit into the catalytic crevice of PphA. Ser49 phosphorylation seems not to be generally conserved in cyanobacteria. In Prochlorococcus marinus, an abundant marine prochlorophyte, the evidences indicate absence of PII phosphorylation (Palinska et al. 2002), in agreement with the lack of a PphA homologue gene (Cyanobase: http:// genome.kazusa.or.jp/cyanobase). In filamentous cyanobacteria, the situation is less clear. No PII phosphorylation could be detected in Nostoc punctiforme extracts but the $N$. punctiforme PII protein could be phosphorylated in vitro by $S$. elongatus cell extracts (Hanson et al. 1998). Mass spectroscopic analysis of the PII protein from Anabaena extracts revealed Tyr51 to be subjected to nitration under diazotrophic conditions while no phosphorylation at Ser49 was detected (Zhang et al. 2007). Absence of PII phosphorylation in the Nostocales is, however, in contrast to the presence of a PphA homologue. Mutation of the PphA homologue gene resulted in altered PII functions in Anabaena (Laurent et al. 2004). In plants, potential PII phosphorylation was investigated in A. thaliana. Its PII protein has conserved the seryl-phosphorylation site, but no phosphorylation could be identified. Recently, the PII protein from Chlamydomonas reinhardtii was characterized. It has a potentially phosphorylatable threonyl residue at the corresponding position, but like in Arabidopsis, protein phosphorylation analysis revealed only non-phosphorylated PII protein (Ermilova et al. 2012). At a first glance, it seems odd that in spite of conservation of this site, phosphorylation of PII seems not to be conserved. However, conservation of this site could be due to its pivotal role in PII-NAGK interaction.

\section{PII-mediated regulation of the arginine pathway in cyanobacteria and plants}

Yeast-two hybrid screening for PII-interaction partners in cyanobacteria and plants using genomic DNA from S. elongatus and A. thaliana identified the enzyme $N$-acetylL-glutamate kinase (NAGK) as a novel PII receptor (Burillo et al. 2004; Heinrich et al. 2004; Sugiyama et al. 2004). In plants and cyanobacteria, NAGK catalyzes the committed step of arginine biosynthesis and in agreement, the enzyme is feedback-inhibited by arginine. In S. elongatus and A. thaliana, PII modulates the catalytic properties of NAGK. Initial experiments (Heinrich et al. 2004; Chen et al. 2006) yielded some inconsistent results due to the use of a non-optimized assay buffer, which impaired 2-OG effects and which lacked a reducing agent, necessary for high NAGK activities (Beez et al. 2009). When tested under optimized conditions, the following common properties between the proteins from $S$. elongatus and A. thaliana became evident (Beez et al. 2009): (1) PII activates the overall catalytic efficiency $\left(k_{\mathrm{cat}} / K_{\mathrm{m}}\right)$ of NAGK, for S. elongatus 8 -fold and for $A$. thaliana 1.5 -fold. In the latter case, activation is mainly an effect on $k_{\text {cat }}$. (2) PII relieves NAGK from arginine feed-back inhibition. It also increases the half maximal inhibitory concentration of arginine $\left(\mathrm{IC}_{50}\right)$ from 20 to $200 \mu \mathrm{M}$ for S. elongatus, and from 1 to $6 \mathrm{mM}$ for A. thaliana NAGK. Arginine inhibits NAGK by increasing the $K_{\mathrm{m}}$ for the substrate NAG, an effect, which is counteracted by PII. (3) 2-OG antagonizes the protection of NAGK by PII from arginine inhibition.

The most prominent differences between NAGK of S. elongatus and A. thaliana are a much higher activity (almost 100-fold) of A. thaliana NAGK and a much higher $\mathrm{IC}_{50}$ towards arginine. In A. thaliana, ATP accelerated complex formation; however, ADP did not negatively affect this process (Beez et al. 2009). By contrast, in 
S. elongatus, ADP lowered the affinity of the PII-NAGK complex formation (Fokina et al. 2011). Therefore, in S. elongatus, PII activation of NAGK activity not only depends on 2-OG but also on the ATP/ADP ratio (Fokina et al. 2011), whereas in A. thaliana, only 2-OG antagonizes NAGK activation by PII (Beez et al. 2009). A remarkable finding was the observation that the A. thaliana PII protein could completely replace $S$. elongatus PII in activating its NAGK. Conversely, S. elongatus PII protein could at least partially activate A. thaliana NAGK. This functional swapping between the cyanobacterial and higher plant PII-NAGK protein pair points out that the fine-tuned interactions between PII and NAGK are extremely conserved. The high degree of similarity was also revealed by the crystal structures of PII-NAGK complexes from S. elongatus (Llacer et al. 2007) and A. thaliana (Mizuno et al. 2007). The NAGK enzyme is a trimer of dimers (each subunit approximately $32 \mathrm{kDa}$ ), assembling into a hexameric toroid with two identical faces. On each side of the toroid one PII trimer attaches (see Fig. 1a) mainly by contacts from the T-loop and by a second interaction surface contributed by the body of the PII protein and B-loop residues (Llacer et al. 2007, 2008; Mizuno et al. 2007). To bind NAGK, the T-loop of PII has to adopt a tightly folded conformation (Llacer et al. 2007; Fokina et al. 2010b). In the 2-OG ligated state, the T-loop is not able to adopt this fold, explaining the antagonistic effect of 2-OG on PII-NAGK complex formation. The tightly folded T-loop inserts into the interdomain crevice of NAGK, a process in which hydrogen bonding interactions of T-loop residue Ser49 (the site of phosphorylation in S. elongatus PII) and an ion pair network organized by Arg45 play a critical role (Llacer et al. 2008). Phosphorylation of Ser49 impairs these interactions and thus prevents NAGK binding in S. elongatus. The pivotal PII residues for NAGK interaction, Arg45, Ser49, and Glu85, as well as the corresponding NAGK residues for these contacts, Glu194, Arg233, Arg254, Ala257, and Gln258, comprise sequence signatures for PII-NAGK interaction unique for most oxygenic phototrophic organisms. Lack of this signature in NAGK sequences from two red algae (Gracilaria tenuistipitata and Cyanidioschyzon merolae) correlates with the apparent absence of PII proteins in these organisms (Llacer et al. 2007). Figure 3 shows an alignment of the C-terminal part of NAGK sequences from oxygenic phototrophs and from various bacteria. Besides the above-mentioned signature sequences, a Cys-Cys pair located between signature residues Arg233 and Ala257 is almost unique for the oxygenic phototrophs. These residues do not directly take part in complex formation. However, it is tempting to speculate that this cysteine-pair could be the cause for the demand of reducing conditions in vitro for the NAGK enzyme activity in cyanobacteria/plants (Beez et al. 2009). Thus, it is likely that NAGK could be redox controlled in oxygenic phototrophs, a mechanism that would allow them to switch off the energy consuming arginine synthesis during light to dark transition.

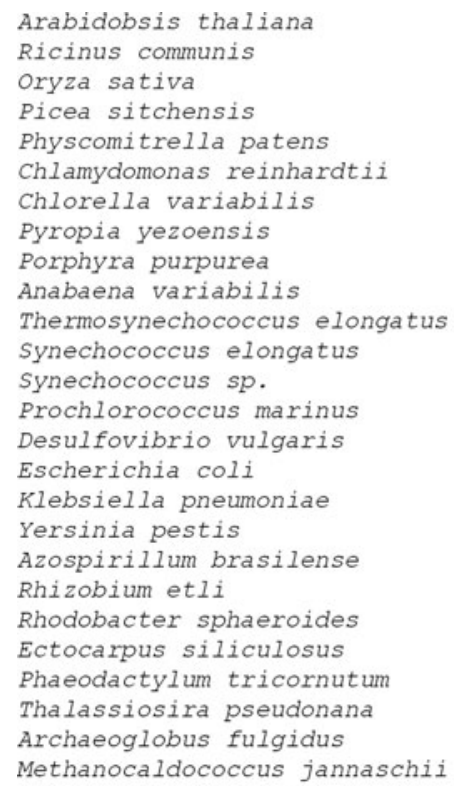

Fig. 3 Multiple sequence alignment of the C-terminal region of NAGK. Representative NAGK sequences from cyanobacteria, green plants, red algae, proteobacteria, archaea, brown algae, and diatoms were aligned using T-Coffee (Di Tommaso et al. 2011) with default

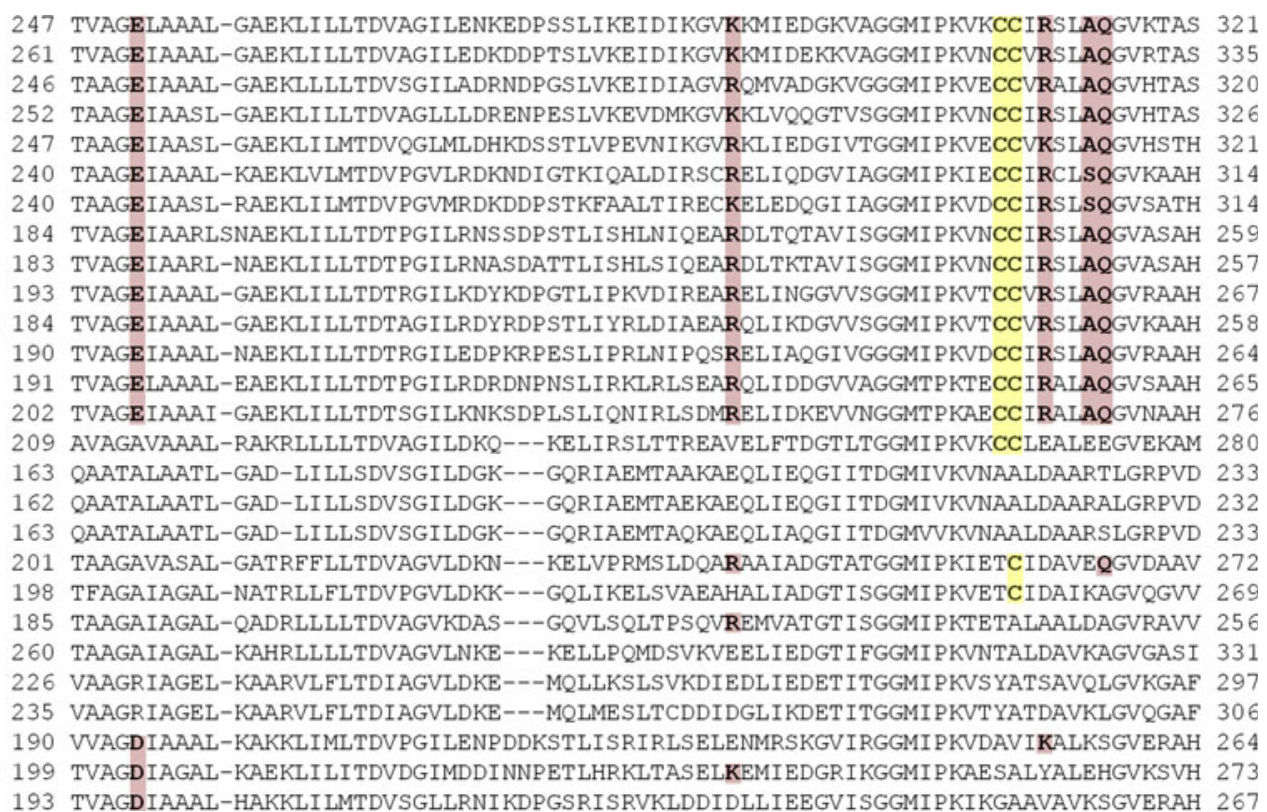

settings. Residues important for interactions with PII are highlighted in salmon. The highly conserved adjacent cysteine residues seen in NAGK sequences of oxygenic phototrophs are shown in yellow with an exception of a proteobacteria Desulfovibrio vulgaris 


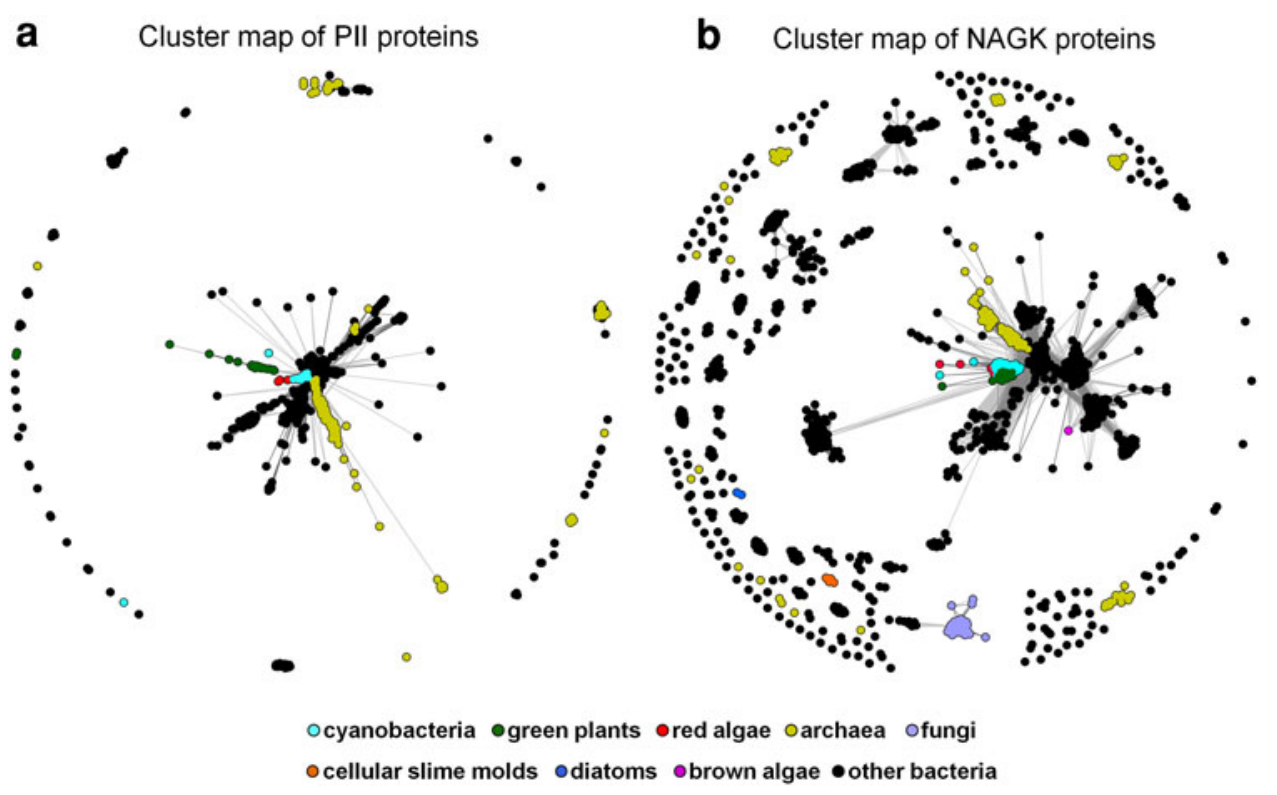

Fig. 4 Cluster map of PII and NAGK proteins. To gather PII and NAGK sequences for cluster analysis, the non-redundant protein sequence database at the NCBI, filtered to a maximum sequence identity of $90 \%$ (nr90), was searched by seeding BLAST (Altschul et al. 1997) with the PII (gi 3885943) and NAGK (gi 332646151) sequences from S. elongatus PCC 6301. All sequence matches with an $E$-value of less than $1 \mathrm{E}-10$ were pooled together. In the next step, the obtained PII and NAGK sequences were clustered separately in CLANS (Frickey and Lupas 2004) based on their all-against-all pairwise similarities as measured by BLAST $P$-values. In the cluster map, dots represent sequences and line coloring reflects BLAST $P$-values; the brighter a line, the lower the $P$-value. Sequences are colored as follows: cyanobacteria-cyan, green

\section{Conservation of PII-NAGK interaction during plastid evolution}

The high degree of conservation in sequence, structure, and function between $S$. elongatus and $A$. thaliana PII-NAGK complexes implies a strong selective pressure for maintaining PII-regulated arginine biosynthesis in the evolution of plastids from an ancestral cyanobacterium. If this is indeed the case, the phylogeny of PII and NAGK sequences should be similar to each other, and should also reflect the evolution of plants. Hitherto studies have only focused on the phylogenetic analysis of the PII superfamily and they have not been conclusive on whether plant PII proteins are of cyanobacterial origin or not (Osanai and Tanaka 2007; Uhrig et al. 2009; Sant'Anna et al. 2009). Since PII proteins are short and highly similar in sequence, reliable inference of their phylogeny is difficult. Also, to our knowledge, a comprehensive phylogenetic analysis of NAGK proteins has not been performed yet. Spurred by this, we decided to revisit the analysis of PII and NAGK proteins using cluster analysis and maximum likelihoodbased phylogenetic reconstruction. In cluster analysis, plants (Viridiplantae)—green, red algae—red, archaea—yellow, fungilight violet, cellular slime molds-orange, diatoms-blue, brown algae-magenta, and other bacteria—black. a 1744 PII sequences obtained using BLAST were clustered at a $P$-value cut-off of $1 \mathrm{E}-33$. At this stringent cut-off, while highly similar sequences remain connected to each other and form central clusters, less similar sequences drift to the periphery of the map. PII sequences from green plants and red algae make their best matches to cyanobacteria, whereas sequences from cyanobacteria are closer to other bacteria. b 1,583 NAGK sequences identified by BLAST are shown. Clustering was carried out at a $P$-value cut-off of $1 \mathrm{E}-$ 83. NAGK sequences from cyanobacteria, green plants, and red algae form a tight cluster in the obtained cluster map

sequences are treated as point masses in a virtual multidimensional space which attract or repel each other depending on the statistical significance of their pairwise sequence similarities. Sequences find their equilibrium position in the map not only by attraction to similar sequences but also by repulsion of different ones. Unlike phylogenetic methods, which have exponential computational complexity and only allow calculation of trees with a few thousand sequences at most, the computational complexity of cluster analyses only increases approximately quadratically with the number of sequences, making calculation of maps with several thousand sequences within a reasonable time possible. In fact, cluster maps become more accurate with an increasing number of sequences as the larger number of pairwise relationships average out the random error arising from simpler pairwise similaritybased comparisons.

In the map of PII proteins (Fig. 4a), the sequences from cyanobacteria (cyan) form a tight cluster that groups together with clusters of sequences from other bacteria (black), whereas sequences from plants (green) and red algae (red) build individual clusters only connected to 


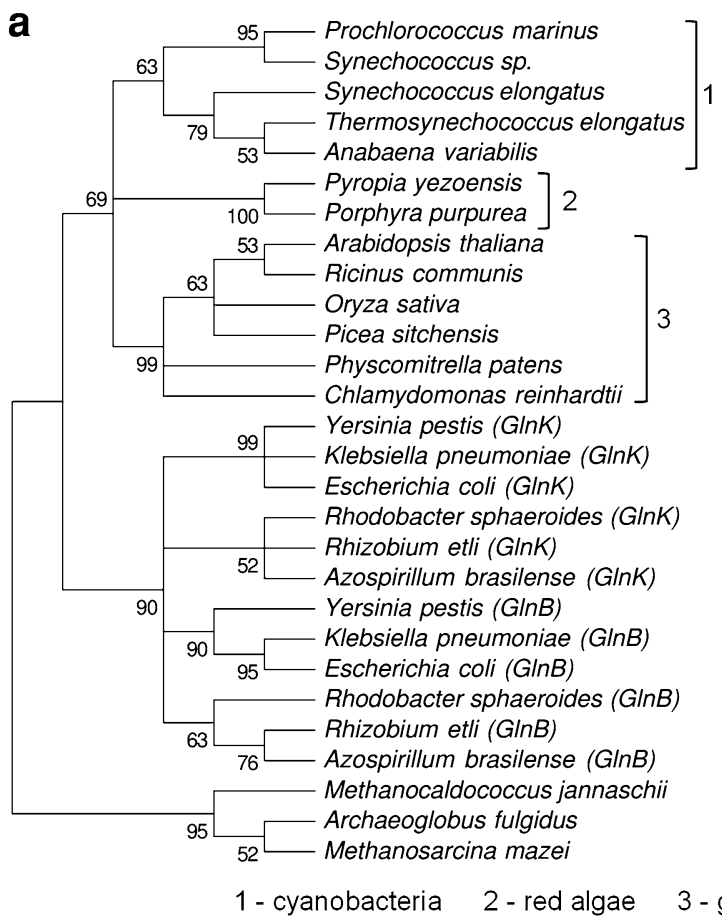

Fig. 5 Molecular phylogenetic analyses of PII and NAGK proteins. For the analysis, representative PII (GlnB), PII-like (GlnK), and NAGK proteins were selected from cyanobacteria, green plants, red algae, $\alpha$-proteobacteria, $\gamma$-proteobacteria, archaea, brown algae, and diatoms. Multiple sequence alignments of PII and NAGK sequences were calculated using T-Coffee (Di Tommaso et al. 2011) with default parameters. Poorly aligned positions and highly divergent regions were removed using Gblocks (Talavera and Castresana 2007). The refined alignments were then used to infer phylogenetic trees in

cyanobacterial sequences (cyan). This suggests that PII proteins of plants and red algae are of cyanobacterial origin, which is in accordance with previous proposals (Osanai and Tanaka 2007; Sant'Anna et al. 2009; Uhrig et al. 2009). While the cyanobacterial cluster is in close proximity to the other bacterial clusters, the plant cluster is further removed from the cyanobacterial cluster. This indicates that PII proteins from cyanobacteria had limited freedom to evolve, but PIIs from plants were altered to adapt to new niches after being acquired from an ancient cyanobacterium. By contrast, in the NAGK map (Fig. 4b), the sequences from cyanobacteria, red algae, and Chloroplastida cluster together indicating an endosymbiotic origin of these proteins. NAGK from the two sequenced diatoms (blue), Thalassiosira pseudonana and Phaeodactylum tricornutum, cluster together with NAGK from alphaproteobacteria. It is possible that in the evolution of heterokonts, which include diatoms and brown algae, an $\operatorname{argB}$ gene (encoding NAGK) was acquired by horizontal gene transfer from an alphaproteobacterium while the original cyanobacterial/plastidal NAGK and PII genes were b

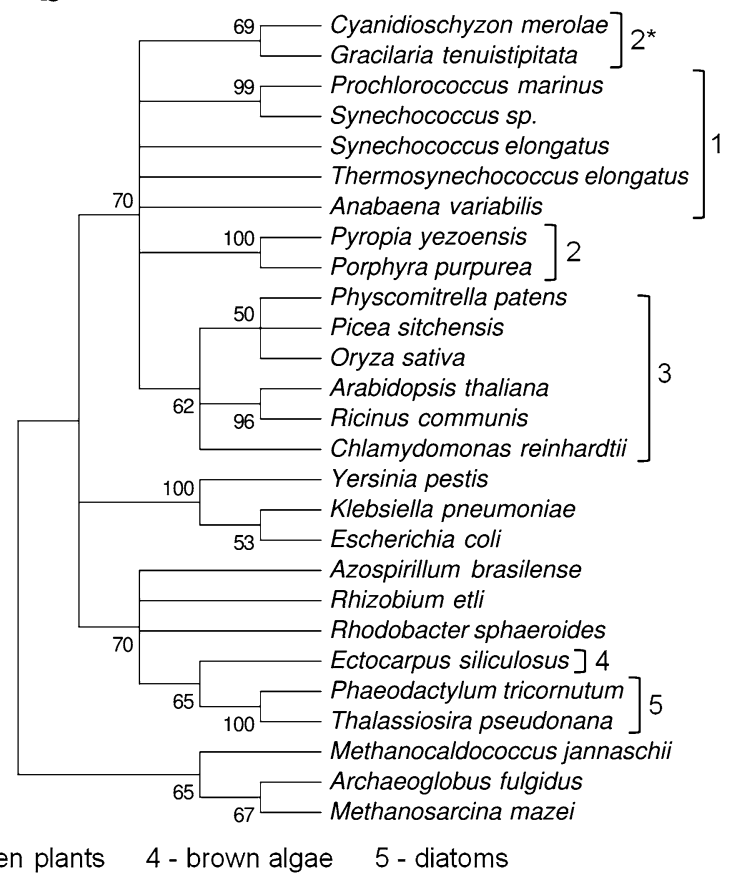

MEGA 5.0 (Tamura et al. 2011), employing the maximum likelihood (ML) method with the WAG model of substitutions and the discrete Gamma distribution model of evolutionary rate variation among sites. The bootstrap consensus trees inferred from 1,000 replicates are shown; branches corresponding to partitions reproduced in less than $50 \%$ bootstrap replicates are collapsed. The phylogenetic tree of PII (a) contains 28 amino acid sequences and that of NAGK (b) consists of 27 sequences

lost. To exclude that this grouping was an artifact of the cluster analysis, we inferred phylogeny of PII (Fig. 5a) and NAGK (Fig. 5b) sequences using maximum likelihoodbased reconstruction. The resulting trees support the phylogenetic grouping exhibited by the cluster maps. Overall, the evolutionary trees of PII and NAGK sequences are in perfect agreement with the assumed evolution of cyanobacteria and of the plant kingdom (Deschamps and Moreira 2009). In support of co-evolution between PII and NAGK, the phylogenetic tree of NAGKs matches the tree of PII sequences, except for the two red algae Gracilaria tenuistipitata and Cyanidioschyzon merolae (denoted as $2 *$ in Fig. 5b), which have lost PII during evolution. Their NAGKs are distantly related to the NAGKs from the other red algae. In these organisms, the loss of PII has released NAGK from the constraint to interact with PII and therefore, their NAGK sequences gained more freedom to evolve. Together, this analysis shows that control of arginine synthesis through PII-dependent signaling was kept under selective pressure in the evolution of plastids of Chloroplastida and most Rhodophyta. 


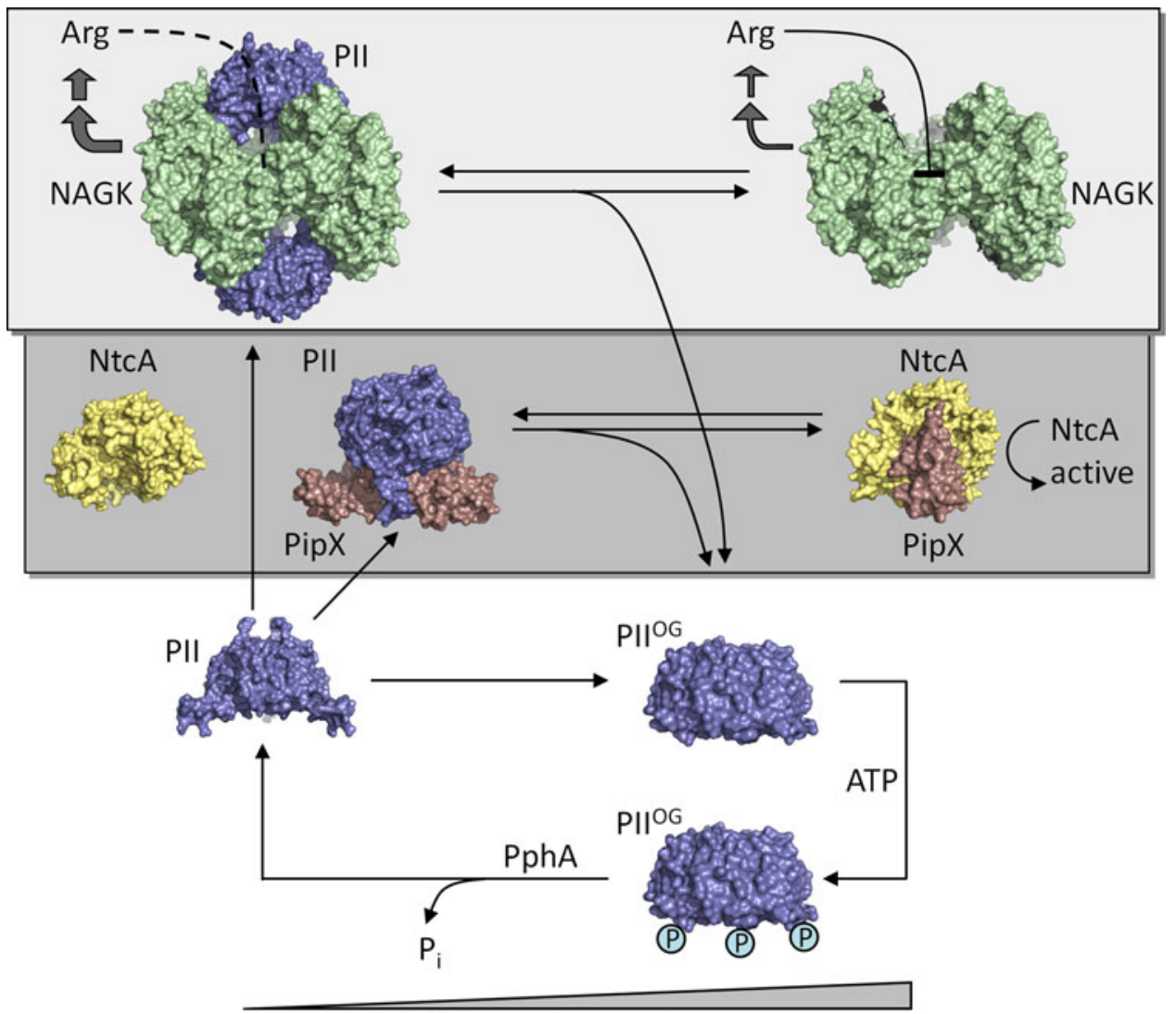

2-OG concentration

Fig. 6 Schematic representation of the 2-oxoglutarate (2-OG) dependent cycle of PII interactions in cyanobacteria under ATP-replete conditions. Under low 2-OG conditions (left), PII (slate) forms a complex with NAGK (light green), the key enzyme of the arginine pathway (top, light gray background), or with PipX (brown), the coactivator of transcription factor NtcA (yellow) (middle part, dark gray background). In complex with PII, NAGK is highly active and protected from tight arginine feedback-inhibition. The NtcA-factor is unable to bind PipX. At increasing 2-OG levels, the PII-complexes dissociate and release 2-OG-ligated PII, which becomes phosphorylated by PII kinase activity. Free NAGK has diminished activity and

\section{Further functions of PII signaling in oxygenic phototrophs}

The complete network of interactions of PII with its binding partners in cyanobacteria (summarized in Fig. 6) has been reviewed previously (Osanai and Tanaka 2007; Forchhammer 2008, 2010). In brief, two major targets of PII regulation have been identified at the molecular level, the transcriptional co-activator PipX and NAGK. PipX, a small protein of approximately 90 amino acids, was shown to be required for full expression of NtcA-activated genes (Espinosa et al. 2006, 2007; Laichoubi et al. 2012). Binding of PipX to NtcA is favored by high 2-OG levels in vitro, whereas 2-OG in concert with Mg-ATP prevents binding of PipX to PII. The structures of PipX-NtcA and PipX-PII complexes have been solved (Llacer et al. 2010; Zhao et al. is tightly feedback-inhibited by arginine. PipX associates with NtcA and activates NtcA-dependent gene expression. When the 2-OG levels drop again, phosphorylated PII has to be dephosphorylated by PII phosphatase PphA before being able to form complexes with NAGK and PipX again. In the chloroplasts of plants, PII-NAGK interaction is conserved, whereas no homologues of PipX, NtcA, and PphA are found. Instead, PII interacts with ACCase (see text for details). PDB ID of structures: 2V5H (PII-NAGK complex), 2XGX (NtcA), 2XG8 (PII-PipX), 2XKO (NtcA-PipX), 1QY7 (free PII), and 2XUL ( $\mathrm{PII}^{2 \mathrm{OG}}$ ). This figure was generated using PyMOL

2010). It is thought that PII binding of PipX tunes down NtcA-dependent gene expression (Espinosa et al. 2007). PipX homologues have been identified in all cyanobacterial genomes, but they are absent in eukaryotes, implying that modulation of gene expression by PII was lost during the endosymbiotic transition of the cyanobacterial ancestor into a chloroplast. A few cyanobacteria possess multiple PII paralogues, and in these cases, the organisms also contain multiple PipX paralogues. In the case of the marine cyanobacterium Synechococcus WH5701, it could be shown that only one of the two PII paralogues activates NAGK and binds to PipX protein; the function of the other PII paralogue is unknown (Laichoubi et al. 2011). A further target of PII was identified as PamA in the cyanobacterium Synechocystis PCC 6803. PamA is a potential transmembrane channel protein of the MscS family with unknown function. 
Mutation of PamA affects the expression of a subset of nitrogen-regulated genes, among them is a sigma-factor required for the expression of sugar-catabolic genes (Osanai et al. 2005). Furthermore, circumstantial evidence suggests that PII is also involved in the control of nitrate/nitrite uptake in S. elongatus (Forchhammer 2010).

The above-mentioned PII receptors are absent in plants; however, a new PII receptor, the chloroplast acetyl-CoA carboxylase (ACCase), a key enzyme in fatty acid synthesis in plastids, was identified by pull-down experiments with $A$. thaliana extracts (Feria Bourrellier et al. 2010). ACCase activity was repressed by PII, but this repression was antagonized by 2-OG and oxaloacetate and to a lesser extent by pyruvate. So far this interaction has not been characterized in great detail and also it needs to be confirmed if this interaction can be generalized for all plants. Nevertheless, the regulation of ACCase by PII represents an intriguing link to carbon storage metabolism. Physiological analyses in A. thaliana support the function of PII in storage metabolism. The PII gene is upregulated in early seed maturation by the transcription factor WRINKLED1 (Baud et al. 2010), and in seeds of PII deficient mutants, a transient increase of fatty acid production and an alteration in fatty acid composition were observed. From these results, a regulatory role of PII in the fine-tuning of fatty acid biosynthesis and partitioning in seeds had been inferred (Baud et al. 2010). Furthermore, in A. thaliana PII mutants, nitrite uptake in chloroplast is enhanced (Ferrario-Mery et al. 2008), resembling the regulatory defect of nitrite/nitrate uptake in cyanobacterial PII mutants (Kloft and Forchhammer 2005). Altogether, the data indicate that during the evolution of plastids, PII lost its primary function of coordinating gene expression through interactions with PipX, but preserved its role in nitrogen (arginine) storage metabolism, and eventually took over fine-tuned regulation of carbon (fatty acid) storage metabolism. Currently, PII is known to play a role in early seed maturation, but it is unclear if it also has roles in other developmental stages of plants. Studies of phylogenetically more ancient plants and of unicellular green algae will be necessary to unravel the roles of PII in the metabolic pathways of Chloroplastida.

Acknowledgments We thank Andrei Lupas for helpful discussions. This study was supported by DFG grant Fo195/9 and by institutional funds from the Max Planck Society.

Conflict of interest The authors declare that they have no conflict of interest.

\section{References}

Adler SP, Purich D, Stadtman ER (1975) Cascade control of Escherichia coli glutamine synthetase. Properties of the PII regulatory protein and the uridylyltransferase-uridylyl-removing enzyme. J Biol Chem 250:6264-6272

Altschul SF, Madden TL, Schaffer AA, Zhang J, Zhang Z, Miller W, Lipman DJ (1997) Gapped BLAST and PSI-BLAST: a new generation of protein database search programs. Nucleic Acids Res 25:3389-3402

Arcondeguy T, Lawson D, Merrick M (2000) Two residues in the T-loop of GlnK determine NifL-dependent nitrogen control of nif gene expression. J Biol Chem 275:38452-38456

Arcondéguy T, Jack R, Merrick M (2001) P(II) signal transduction proteins, pivotal players in microbial nitrogen control. Microbiol Mol Biol Rev 65:80-105

Arnesano F, Banci L, Benvenuti M, Bertini I, Calderone V, Mangani S, Viezzoli MS (2003) The evolutionarily conserved trimeric structure of CutA1 proteins suggests a role in signal transduction. J Biol Chem 278:45999-46006

Atkinson MR, Kamberov ES, Weiss RL, Ninfa AJ (1994) Reversible uridylylation of the Escherichia coli PII signal transduction protein regulates its ability to stimulate the dephosphorylation of the transcription factor nitrogen regulator I (NRI or NtrC). J Biol Chem 269:28288-28293

Baud S, Feria Bourrellier AB, Azzopardi M, Berger A, Dechorgnat J, Daniel-Vedele F, Lepiniec L, Miquel M, Rochat C, Hodges M, Ferrario-Mery S (2010) PII is induced by WRINKLED1 and fine-tunes fatty acid composition in seeds of Arabidopsis thaliana. Plant J 64:291-303

Beez S, Fokina O, Herrmann C, Forchhammer K (2009) $N$-acetyl-Lglutamate kinase (NAGK) from oxygenic phototrophs: $\mathrm{P}(\mathrm{II})$ signal transduction across domains of life reveals novel insights in NAGK control. J Mol Biol 389:748-758

Burillo S, Luque I, Fuentes I, Contreras A (2004) Interactions between the nitrogen signal transduction protein PII and $N$-acetyl glutamate kinase in organisms that perform oxygenic photosynthesis. J Bacteriol 186:3346-3354

Cheah E, Carr PD, Suffolk PM, Vasudevan SG, Dixon NE, Ollis DL (1994) Structure of the Escherichia coli signal transducing protein PII. Structure 2:981-990

Chen YM, Ferrar TS, Lohmeier-Vogel EM, Morrice N, Mizuno Y, Berenger B, Ng KK, Muench DG, Moorhead GB (2006) The PII signal transduction protein of Arabidopsis thaliana forms an arginine-regulated complex with plastid $\mathrm{N}$-acetyl glutamate kinase. J Biol Chem 281:5726-5733

Cho Y, Sharma V, Sacchettini JC (2003) Crystal structure of ATP phosphoribosyltransferase from Mycobacterium tuberculosis. J Biol Chem 278:8333-8339

Deschamps P, Moreira D (2009) Signal conflicts in the phylogeny of the primary photosynthetic eukaryotes. Mol Biol Evol 26:2745-2753

Di Tommaso P, Moretti S, Xenarios I, Orobitg M, Montanyola A, Chang JM, Taly JF, Notredame C (2011) T-Coffee: a web server for the multiple sequence alignment of protein and RNA sequences using structural information and homology extension. Nucleic Acids Res 39 (Web Server issue):W13-17

Ermilova E, Lapina T, Zalutskaya Z, Minaeva E, Fokina O, Forchhammer K (2012) PII Signal Transduction Protein in Chlamydomonas reinhardtii: localization and Expression Pattern. Protist. doi:10.1016/j.protis.2012.04.002

Espinosa J, Forchhammer K, Burillo S, Contreras A (2006) Interaction network in cyanobacterial nitrogen regulation: PipX, a protein that interacts in a 2-oxoglutarate dependent manner with PII and NtcA. Mol Microbiol 61:457-469

Espinosa J, Forchhammer K, Contreras A (2007) Role of the Synechococcus PCC 7942 nitrogen regulator protein PipX in NtcA-controlled processes. Microbiology 153:711-718

Feria Bourrellier AB, Valot B, Guillot A, Ambard-Bretteville F, Vidal J, Hodges M (2010) Chloroplast acetyl-CoA carboxylase activity 
is 2-oxoglutarate-regulated by interaction of PII with the biotin carboxyl carrier subunit. Proc Natl Acad Sci USA 107:502-507

Ferrario-Mery S, Meyer C, Hodges M (2008) Chloroplast nitrite uptake is enhanced in Arabidopsis PII mutants. FEBS Lett 582:1061-1066

Fokina O, Chellamuthu VR, Forchhammer K, Zeth K (2010a) Mechanism of 2-oxoglutarate signaling by the Synechococcus elongatus PII signal transduction protein. Proc Natl Acad Sci USA 107:19760-19765

Fokina O, Chellamuthu VR, Zeth K, Forchhammer K (2010b) A novel signal transduction protein $\mathrm{P}(\mathrm{II})$ variant from Synechococcus elongatus PCC 7942 indicates a two-step process for NAGK-P(II) complex formation. J Mol Biol 399:410-421

Fokina O, Herrmann C, Forchhammer K (2011) Signal-transduction protein P(II) from Synechococcus elongatus PCC 7942 senses low adenylate energy charge in vitro. Biochem J 440:147-156

Forchhammer K (2008) P(II) signal transducers: novel functional and structural insights. Trends Microbiol 16:65-72

Forchhammer K (2010) The network of P(II) signalling protein interactions in unicellular cyanobacteria. Adv Exp Med Biol 675:71-90

Forchhammer K, Hedler A (1997) Phosphoprotein PII from cyanobacteria-analysis of functional conservation with the PII signaltransduction protein from Escherichia coli. Eur J Biochem 244:869-875

Forchhammer K, Tandeau de Marsac N (1994) The PII protein in the cyanobacterium Synechococcus sp. strain PCC 7942 is modified by serine phosphorylation and signals the cellular $\mathrm{N}$-status. J Bacteriol 176:84-91

Frickey T, Lupas A (2004) CLANS: a Java application for visualizing protein families based on pairwise similarity. Bioinformatics 20:3702-3704

Hanson TE, Forchhammer K, Tandeau de Marsac N, Meeks JC (1998) Characterization of the glnB gene product of Nostoc punctiforme strain ATCC 29133: glnB or the PII protein may be essential. Microbiology 144:1537-1547

Heinrich A, Maheswaran M, Ruppert U, Forchhammer K (2004) The Synechococcus elongatus $\mathrm{P}$ signal transduction protein controls arginine synthesis by complex formation with $\mathrm{N}$-acetyl-L-glutamate kinase. Mol Microbiol 52:1303-1314

Hsieh MH, Lam HM, van de Loo FJ, Coruzzi G (1998) A PII-like protein in Arabidopsis: putative role in nitrogen sensing. Proc Natl Acad Sci USA 95:13965-13970

Huergo LF, Chandra G, Merrick M (2012) PII signal transduction proteins: nitrogen regulation and beyond. FEMS Microbiol Rev. doi:10.1111/j.1574-6976.2012.00351.x

Irmler A, Forchhammer K (2001) A PP2C-type phosphatase dephosphorylates the PII signaling protein in the cyanobacterium Synechocystis PCC 6803. Proc Natl Acad Sci USA 98:12978-12983

Jiang P, Ninfa AJ (2011) A source of ultrasensitivity in the glutamine response of the bicyclic cascade system controlling glutamine synthetase adenylylation state and activity in Escherichia coli. Biochemistry 50:10929-10940

Kinch LN, Grishin NV (2002) Expanding the nitrogen regulatory protein superfamily: homology detection at below random sequence identity. Proteins 48:75-84

Kloft N, Forchhammer K (2005) Signal transduction protein PII phosphatase PphA is required for light-dependent control of nitrate utilization in Synechocystis sp. strain PCC 6803. J Bacteriol 187:6683-6690

Laichoubi KB, Beez S, Espinosa J, Forchhammer K, Contreras A (2011) The nitrogen interaction network in Synechococcus WH5701, a cyanobacterium with two PipX and two P(II)-like proteins. Microbiology 157:1220-1228
Laichoubi KB, Espinosa J, Castells MA, Contreras A (2012) Mutational analysis of the cyanobacterial nitrogen regulator PipX. PLoS ONE 7(4):e35845. doi:10.1371/journal.pone. 0035845

Laurent S, Forchhammer K, Gonzalez L, Heulin T, Zhang CC, Bedu S (2004) Cell-type specific modification of PII is involved in the regulation of nitrogen metabolism in the cyanobacterium Anabaena PCC 7120. FEBS Lett 576:261-265

Leigh JA, Dodsworth JA (2007) Nitrogen regulation in bacteria and archaea. Annu Rev Microbiol 61:349-377

Litz C, Helfmann S, Gerhardt S, Andrade SL (2011) Structure of GlnK1, a signalling protein from Archaeoglobus fulgidus. Acta Crystallogr Sect F Struct Biol Cryst Commun 67:178-181

Llacer JL, Contreras A, Forchhammer K, Marco-Marin C, Gil-Ortiz F, Maldonado R, Fita I, Rubio V (2007) The crystal structure of the complex of PII and acetylglutamate kinase reveals how PII controls the storage of nitrogen as arginine. Proc Natl Acad Sci USA 104:17644-17649

Llacer JL, Fita I, Rubio V (2008) Arginine and nitrogen storage. Curr Opin Struct Biol 18:673-681

Llacer JL, Espinosa J, Castells MA, Contreras A, Forchhammer K, Rubio V (2010) Structural basis for the regulation of NtcAdependent transcription by proteins PipX and PII. Proc Natl Acad Sci USA 107:15397-15402

Lohkamp B, McDermott G, Campbell SA, Coggins JR, Lapthorn AJ (2004) The structure of Escherichia coli ATP-phosphoribosyltransferase: identification of substrate binding sites and mode of AMP inhibition. J Mol Biol 336:131-144

Luque I, Forchhammer K (2007) Nitrogen assimilation and C/N balance sensing. In: Herrero A, Flores E (eds) The Cyanobacteria: Molecular Biology. Genomics and Evolution Caister Academic Press, Norfolk, pp 335-382

Maier S, Schleberger P, Lu W, Wacker T, Pfluger T, Litz C, Andrade SL (2011) Mechanism of disruption of the Amt-GlnK complex by P(II)-mediated sensing of 2-oxoglutarate. PLoS ONE 6:e26327. doi:10.1371/journal.pone.0026327

Masepohl B, Forchhammer K (2007) Regulatory cascades to express nitrogenase. In: Bothe $\mathrm{H}$, Ferguson $\mathrm{S}$, Newton WE (eds) Molecular biology, biochemistry and applied aspects of the nitrogen cycle. Elsevier B.V, Amsterdam, pp 131-145

Mizuno YB, Moorhead GB, Ng KK (2007) Crystal structure of Arabidopsis PII reveals novel structural elements unique to plants. Biochemistry 46:1477-1483

Ninfa AJ, Atkinson MR (2000) PII signal transduction proteins. Trends Microbiol 8:172-179

Osanai T, Tanaka K (2007) Keeping in touch with PII: PII-interacting proteins in unicellular cyanobacteria. Plant Cell Physiol 48:908-914

Osanai T, Kanesaki Y, Nakano T, Takahashi H, Asayama M, Shirai M, Kanehisa M, Suzuki I, Murata N, Tanaka K (2005) Positive regulation of sugar catabolic pathways in the cyanobacterium Synechocystis sp. PCC 6803 by the group 2 sigma factor sigE. J Biol Chem 280:30653-30659

Palinska KA, Laloui W, Bedu S, Loiseaux-de Goer S, Castets AM, Rippka R, Tandeau de Marsac N (2002) The signal transducer $\mathrm{P}$ (II) and bicarbonate acquisition in Prochlorococcus marinus PCC 9511, a marine cyanobacterium naturally deficient in nitrate and nitrite assimilation. Microbiology 148:2405-2412

Perrier AL, Cousin X, Boschetti N, Haas R, Chatel JM, Bon S, Roberts WL, Pickett SR, Massoulie J, Rosenberry TL, Krejci E (2000) Two distinct proteins are associated with tetrameric acetylcholinesterase on the cell surface. J Biol Chem 275:34260-34265

Radchenko M, Merrick M (2011) The role of effector molecules in signal transduction by PII proteins. Biochem Soc Trans 39:189-194 
Ruppert U, Irmler A, Kloft N, Forchhammer K (2002) The novel protein phosphatase PphA from Synechocystis PCC 6803 controls dephosphorylation of the signalling protein PII. Mol Microbiol 44:855-864

Saikatendu KS, Zhang X, Kinch L, Leybourne M, Grishin NV, Zhang H (2006) Structure of a conserved hypothetical protein SA1388 from $S$. aureus reveals a capped hexameric toroid with two PII domain lids and a dinuclear metal center. BMC Struct Biol 6:1. doi:10.1186/1472-6807-6-27

Sant'Anna FH, Trentini DB, de Souto Weber S, Cecagno R, da Silva SC, Schrank IS (2009) The PII superfamily revised: a novel group and evolutionary insights. J Mol Evol 68:322-336

Schlicker C, Fokina O, Kloft N, Grune T, Becker S, Sheldrick GM, Forchhammer K (2008) Structural analysis of the PP2C phosphatase tPphA from Thermosynechococcus elongatus: a flexible flap subdomain controls access to the catalytic site. J Mol Biol 376:570-581

Smith CS, Weljie AM, Moorhead GB (2003) Molecular properties of the putative nitrogen sensor PII from Arabidopsis thaliana. Plant J 33:353-360

Su J, Forchhammer K (2011) Determinants for substrate specificity of the bacterial PP2C protein phosphatase tPphA from Thermosynechococcus elongatus. FEBS J. doi:10.1111/j.1742-4658. 2011.08466.x

$\mathrm{Su}$ J, Forchhammer K (2012) The Role of Arg13 in Protein Phosphatase M tPphA from Thermosynechococcus elongatus. Enzyme Res 2012:272706. doi:10.1155/2012/272706

Su J, Schlicker C, Forchhammer K (2011) A third metal is required for catalytic activity of the signal-transducing protein phosphatase M tPphA. J Biol Chem 286:13481-13488

Sugiyama K, Hayakawa T, Kudo T, Ito T, Yamaya T (2004) Interaction of $N$-acetylglutamate kinase with a PII-like protein in rice. Plant Cell Physiol 45:1768-1778
Talavera G, Castresana J (2007) Improvement of phylogenies after removing divergent and ambiguously aligned blocks from protein sequence alignments. Syst Biol 56:564-577

Tamura K, Peterson D, Peterson N, Stecher G, Nei M, Kumar S (2011) MEGA5: molecular evolutionary genetics analysis using maximum likelihood, evolutionary distance, and maximum parsimony methods. Mol Biol Evol 28:2731-2739

Thomas G, Coutts G, Merrick M (2000) The $g \ln K a m t$ B operon. A conserved gene pair in prokaryotes. Trends Genet 16:11-14

Truan D, Huergo LF, Chubatsu LS, Merrick M, Li XD, Winkler FK (2010) A new P(II) protein structure identifies the 2-oxoglutarate binding site. J Mol Biol 400:531-539

Uhrig RG, Ng KK, Moorhead GB (2009) PII in higher plants: a modern role for an ancient protein. Trends Plant Sci 14:505-511

$\mathrm{Xu}$ Y, Cheah E, Carr PD, van Heeswijk WC, Westerhoff HV, Vasudevan SG, Ollis DL (1998) GlnK, a PII-homologue: structure reveals ATP binding site and indicates how the T-loops may be involved in molecular recognition. $\mathrm{J}$ Mol Biol 282:149-165

Zeth K, Fokina O, Forchhammer K (2012) An engineered PII protein variant that senses a novel ligand: atomic resolution structure of the complex with citrate. Acta Cryst D68:901-908

Zhang Y, Pu H, Wang Q, Cheng S, Zhao W, Zhao J (2007) PII is important in regulation of nitrogen metabolism but not required for heterocyst formation in the Cyanobacterium Anabaena sp. PCC 7120. J Biol Chem 282:33641-33648

Zhao MX, Jiang YL, Xu BY, Chen Y, Zhang CC, Zhou CZ (2010) Crystal structure of the cyanobacterial signal transduction protein PII in complex with PipX. J Mol Biol 402:552-559 